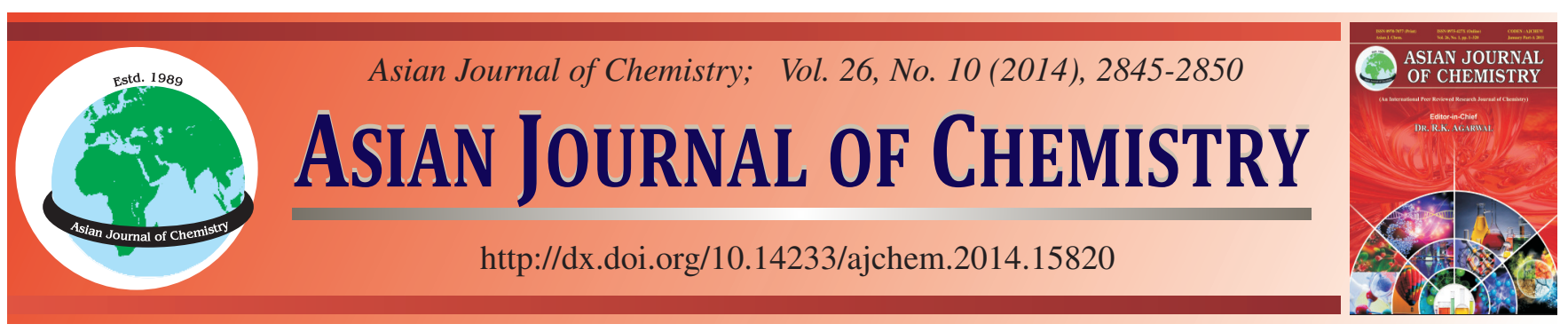

\title{
Synthesis of Copper(I) Oxide Hollow Microspheres with the Assistance of Polyoxyethylenenonylphenylether
}

\author{
Kanda Wongwailikhit ${ }^{*}$ and Nutcha SRIPOOMWATtana
}

Department of Chemistry, Faculty of Science, Rangsit University, 52/374 Muang-Ake Village, Phaholyothin Road, Patumtani 12000, Thailand

*Corresponding author: E-mail: kanda@rsu.ac.th; kanda_wong@yahoo.com

Hollow copper(I) oxide microspheres were prepared by using polyoxyethylene (9) nonylphenyl ether (PONPE-9) as a shape controlling agent. Copper(II) sulphate and ascorbic acid were used as the starting and reducing agents, respectively. The reaction was conducted under sonication without rigid templates at a controlled temperature of $40{ }^{\circ} \mathrm{C}$. The product morphology was found to be affected by solution $\mathrm{pH}$ (from 3-11) and hollow spheres could be prepared only at neutral $\mathrm{pH}$. The outer diameter of the spheres was $600-1,500 \mathrm{~nm}$ and shell thickness $50-70 \mathrm{~nm}$. Similar results were obtained by using PONPE-7 or PONPE-12 but not with other commercial surfactants such as sodium dodecylsulphate, cetryltrimethylbromide and dioctylsodiumsulfosuccinate (AOT). The particle samples were characterized by X-ray powder diffraction, scanning electron microscopy, transmission electron microscopy and electron dispersion spectroscopy. A possible formation mechanism of hollow sphere $\mathrm{Cu}_{2} \mathrm{O}$ produced was also discussed.

Keywords: Microstructure, Cluster assembly, Chemical synthesis.

\section{INTRODUCTION}

Copper(I) oxide particles have been attracting a great deal of interest in a number of research areas because of its unique material properties and potential applications in semiconductor electronics ${ }^{1}$, catalysis and photocatalysis ${ }^{2,3}$, conductive inks ${ }^{4}$, gas sensing, etc. ${ }^{5}$. To enhance its performance, a number of studies have been carried out to develop new and effective methods for the synthesis and manipulation of $\mathrm{Cu}_{2} \mathrm{O}$ particles of controlled sizes and shapes ${ }^{6-8}$. This has in fact been the focus of ongoing research ${ }^{9-14}$, although mass production of nano$\mathrm{Cu}_{2} \mathrm{O}$ has been commercialized worldwide. Among these, $\mathrm{Cu}_{2} \mathrm{O}$ spheres with hollow interiors are particularly interesting, because of the unique properties such as low density, high specific surface area and cage coverage with good permeation. Recently, the synthesis of nano- and micro-sized $\mathrm{Cu}_{2} \mathrm{O}$ hollow spheres of various methodologies has been published ${ }^{15,16}$. In most of these studies the self-assembly of surfactants or long chain organic compounds was exploited to facilitate the formation of spherical particles. For instance, Chen et al. ${ }^{17}$ used gelatin as a soft-template and was able to synthesize $\mathrm{Cu}_{2} \mathrm{O}$ hollow spheres with an average size of $150 \mathrm{~nm}$. Xu et al. ${ }^{18}$ designed an emulsion supporting system to prepare submicron $\mathrm{Cu}_{2} \mathrm{O}$ hollow spheres with the diameter ranging from 600$1200 \mathrm{~nm}$. Chang et al. ${ }^{19}$ used a solvothermal method with N,Ndimethylformamide (DMF) as a reductant and produced $\mathrm{Cu}_{2} \mathrm{O}$ of 100-120 nm in diameter by heating in a Teflon-lined stainless steel autoclave at $150-180{ }^{\circ} \mathrm{C}$. Sui et al..$^{20}$ used poly(vinylpyrrolidone) (PVP) in a reactor at $90{ }^{\circ} \mathrm{C}$ to produce hollow $\mathrm{Cu}_{2} \mathrm{O}$ of 400-700 nm in diameter. With the help of Tween-60 at room temperature, $\mathrm{Xu}$ and co-worker made $\mathrm{Cu}_{2} \mathrm{O}$ hollow spheres of $500 \mathrm{~nm}$ in diameter ${ }^{21}$. In other studies, multishell hollow copper(I) oxide particles were also prepared. For example $\mathrm{Xu}$ and $\mathrm{Wang}^{22}$; and Zhang et al. ${ }^{23}$ developed a procedure to prepare $\mathrm{Cu}_{2} \mathrm{O}$ particles with multilayer shells with the assistance of cetyltrimethylammonium bromide (CTAB) in ethanol and glutamic acid. In these earlier studies surfactants are used extensively as a shape controller. However, none of these surfactants exhibits a phenyl moiety like polyoxyalkylphenylether (PONPE). This is the primary motivation of our present work.

In this study, PONPE was used as the reaction media to support the formation of $\mathrm{Cu}_{2} \mathrm{O}$ hollow spheres. The reaction was carried out at low temperatures in a single step without the use of any rigid template. It is likely that it may be used for the preparation of other functional materials as well.

\section{EXPERIMENTAL}

Copper sulphate pentahydrate and ascorbic acid were of analytical grade purchased from J.T.Baker Co. Ltd. Polyoxyethylene (7) nonylphenyl ether (PONPE-7), polyoxyethylene (9) nonylphenyl ether (PONPE-9) and polyoxyethylene (12) 
nonylphenyl ether (PONPE-12) were purchased from Aldrich chemicals. Sodium dodecylsulphate (SDS), cetryltrimethyl bromide (CTAB) and dioctyl sodium sulfosuccinate, aerosol OT (AOT) were purchased from Fluka Chemicals. Absolute ethanol was obtained from Mallinckrodt Chemicals. All reagents were used as received without further purification. Water was doubly deionized and distilled.

Methods: The reactions were conducted by mixing $10 \mathrm{~mL}$ of $50 \mathrm{mM}$ surfactants with $1 \mathrm{~mL}$ of $0.5 \mathrm{M} \mathrm{CuSO}_{4} \cdot \mathrm{pH}$ of the solution was adjusted with $0.1 \mathrm{M} \mathrm{H}_{2} \mathrm{SO}_{4}$ or $0.1 \mathrm{M} \mathrm{NaOH}$, into which was added $250 \mu \mathrm{L}$ of $1.0 \mathrm{M}$ ascorbic acid. The mixed solution was then subjected to a temperature controllable sonicator for $2 \mathrm{~h}$ at $40{ }^{\circ} \mathrm{C}$. The precipitates were collected, washed extensively with distilled water and absolute ethanol and finally dried at $100{ }^{\circ} \mathrm{C}$ overnight.

Characterization: The surface morphology and size of the products were examined by scanning electron microscopic measurements with a JEOL model JSM 6301-F microscope mounted with an electron dispersion spectroscopy (EDS) accessory. The phase and the crystallographic structure of the products were characterized by X-ray diffraction analysis (Bruker AXS, Model D8 Advance) using $\mathrm{CuK}_{\alpha}$ at $40 \mathrm{kV}, 30$ $\mathrm{mA}$ and1.54056 ̊. Transmission electron microscopic (TEM) images were acquired with a Hitachi Model H-800 transmission electron microscope operated at $200 \mathrm{kV}$.

\section{RESULTS AND DISCUSSION}

Effect of pH on product morphology: The synthesis was carried out at three different $\mathrm{pHs}$ of 3, 7 and 11 in the presence of PONPE-9. At $\mathrm{pH}=3$ a red-brown precipitate was obtained at the bottom of the reaction container, while a yellow brown precipitate was observed in both the neutral and basic solutions. The different colour appearance of the precipitates suggested a variation of the molecular structure of the products. The morphology of the as-prepared precipitates were then studied by SEM measurements and the results are shown in Fig. 1. The low-magnification images reveal different morphologies of the samples prepared at three different pHs. From Fig. 1(a) it can be seen that the sample prepared at $\mathrm{pH}=3$ exhibited the coexistence of various crystal morphologies such as triangles (arrow 1), rods (arrow 2), dodecahedra (arrow 3) as well as many other unidentified geometric shapes. For the sample prepared at neutral pH in Fig. 1(b), one can see the spherical morphology with a small size distribution between 0.6 and $1.5 \mu$. In contrast, for the product produced at $\mathrm{pH}=11 \mathrm{in} \mathrm{Fig.}$ $1(\mathrm{c})$, only the agglomeration of very small irregular-shape particles were observed.

Images at higher magnification were depicted in Fig. 2. The results showed good reproducibility of our reaction procedure. The sample obtained at $\mathrm{pH}=3$ in Fig. 2(a) exhibits the
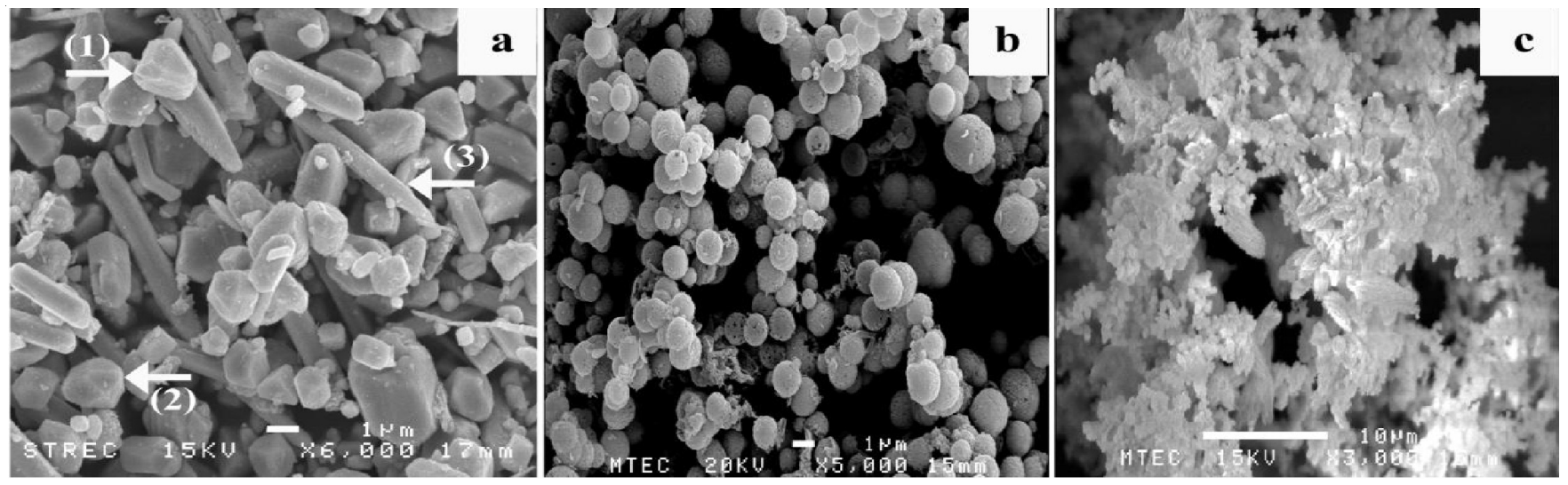

Fig. 1. Low magnification of SEM images of precipitates synthesized in the presence PONPE- 9 at three different pHs: $\left[\mathrm{CuSO}_{4}\right]=0.045 \mathrm{M},\left[\mathrm{C}_{6} \mathrm{H}_{8} \mathrm{O}_{6}\right]=0.02$ $\mathrm{M}[\mathrm{PONPE}-9]=0.04$ molal, $40^{\circ} \mathrm{C}$. (a) $\mathrm{pH} 3$ (b) $\mathrm{pH} 7$ (c) $\mathrm{pH} 11$
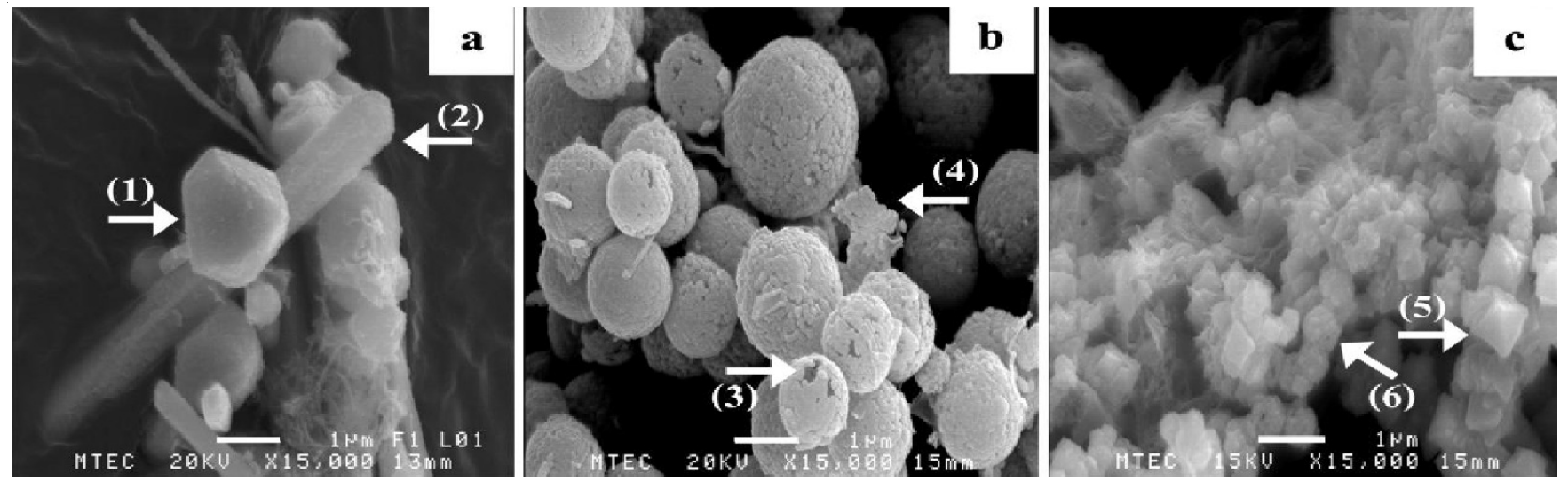

Fig. 2. SEM images at 15,000 magnification of precipitates synthesized in the presence PONPE-9 at three different pHs: $\left[\mathrm{CuSO}_{4}\right]=0.045 \mathrm{M}_{,}\left[\mathrm{C}_{6} \mathrm{H}_{8} \mathrm{O}_{6}\right]=$ $0.02 \mathrm{M}[\mathrm{PONPE}-9]=0.04$ molal, $40{ }^{\circ} \mathrm{C}$. (a) $\mathrm{pH} 3$ (b) $\mathrm{pH} 7$ (c) $\mathrm{pH} 11$ 
appearance of many geometrical shapes, e.g., triangles (arrow 1 ), rods (arrow 2) and others. The sample at $\mathrm{pH}=7$ shows an interesting spherical morphology with a porous surface (Fig. 2(b)). Some of the spheres exhibit a hollow interior (arrow 3); and, some display a sheet-like crystal structure (arrow 4), possibly an incomplete curved sheet. These observations suggest that the microspheres were likely formed by a self-encircling process resulting in a hollow structure. Fig. 2(c) shows the image of the product prepared at $\mathrm{pH}=11$. It shows a picture of the coexistence of octahedra (arrow 5) and spheres (arrow $6)$.

The shape distinction of the products prepared at different $\mathrm{pHs}$ indicates that $\mathrm{pH}$ of the solutions is an important parameter in controlling the product morphology $y^{7,12,20,24}$. It should be mentioned here that copper(II) ion can be reduced in more than one pathway in the presence of hydrogen ion $\left(\mathrm{H}^{+}\right)$and hydroxide ion $\left(\mathrm{OH}^{-}\right)$, leading to the generation of different products.

Fig. 3(a-b) depict the TEM images of the sample prepared at $\mathrm{pH}=7$ which clearly show the existence of a hollow interior with a diameter of 500-600 $\mathrm{nm}$ and the spheres are roughly spherical in shape and possess a thin shell layer with the
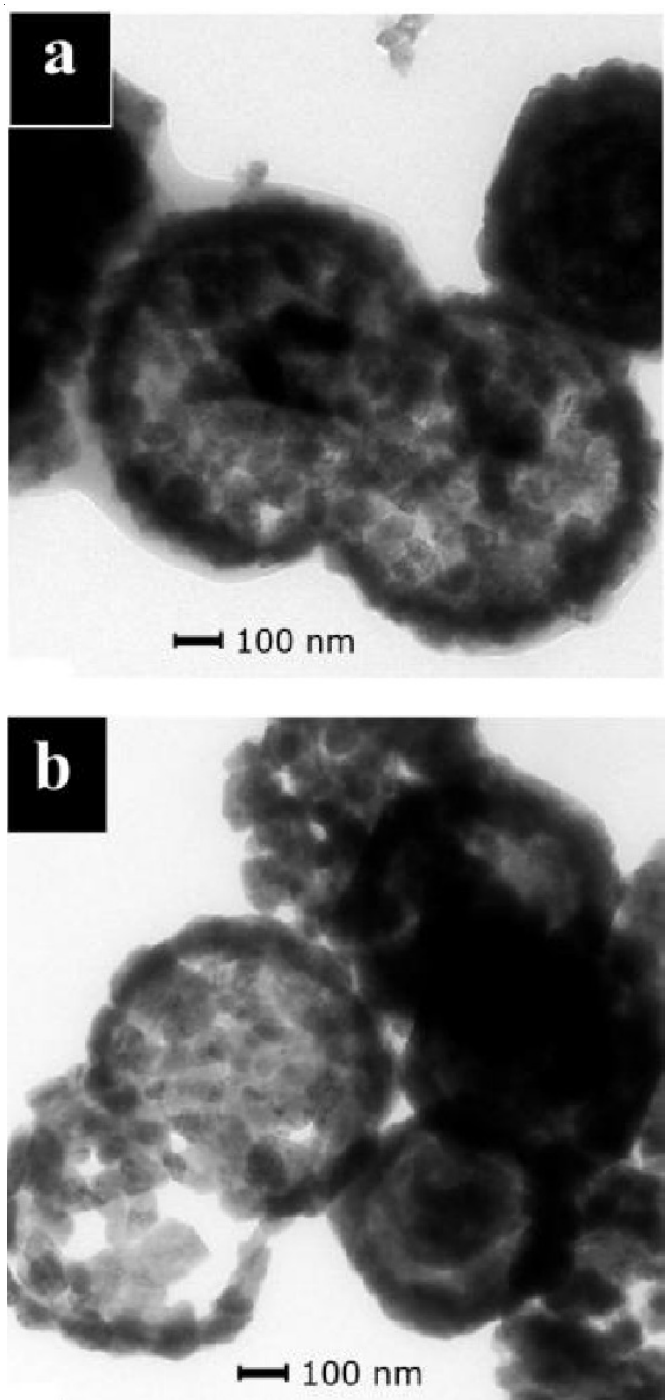

Fig. 3. TEM of the image of precipitate prepared with the assistance of PONPE-9 at $\mathrm{pH} 7$ thickness of 50-70 nm. Also the sphere shell appeared to be composed of many tiny clusters attached each together. With these observations, it is likely that the clusters were initiated by the formation of nuclei at the peripheral sites of the micellelike templates formed by PONPE. Once the nuclei have grown, they become tiny clusters which attach to each other around the outer shell of the micelles. If the clusters fail to aggregate at some spots, the vacancy yields an incomplete spherical wall as shown in Fig. 3(b). The coalescence of nanosized clusters forming spherical walls can be seen in SEM measurements (Fig. 4). That is, hollow sphere crystals could be formed with the assistance of PONPE-9 via the self assembly process. With the porous particle shells, the hollow spheres possess a high surface area. The mechanism of the formation of hollow spheres via this self-assembly process is proposed at the final part of this report.

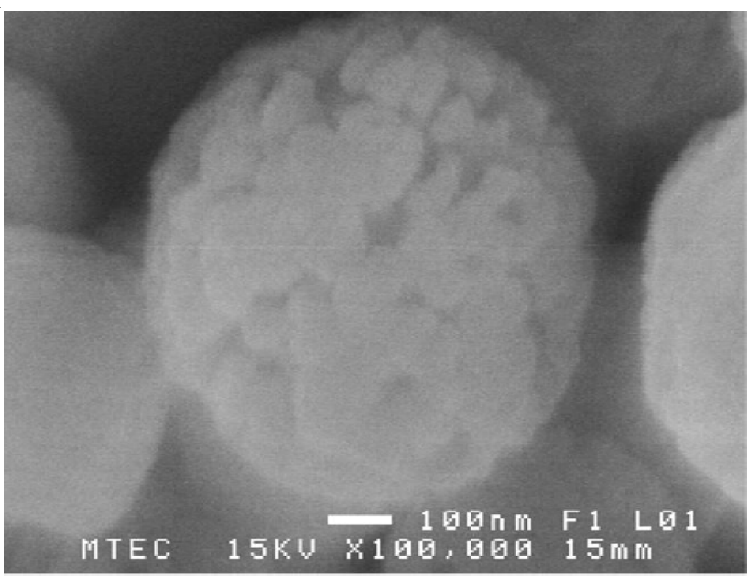

Fig. 4. SEM image at 100,000 magnification of $\mathrm{Cu}_{2} \mathrm{O}$ produced with the assistance of PONPE-9

The element compositions of the products were then examined by EDS analysis and their spectra are shown in Fig. 5. It is clearly shown that in Fig. 5(a) only pure copper metal was produced in the sample prepared at $\mathrm{pH}=3$. This suggested that copper(II) ion was reduced by ascorbic acid under acid conditions and yielded pure copper metal. The reaction could be described as:

$$
\mathrm{C}_{6} \mathrm{H}_{8} \mathrm{O}_{6}(\mathrm{aq})+\mathrm{Cu}^{2+}(\mathrm{aq}) \rightarrow \mathrm{C}_{6} \mathrm{H}_{6} \mathrm{O}_{6}(\mathrm{aq})+\mathrm{Cu}^{\mathrm{o}}(\mathrm{s})+2 \mathrm{H}^{+}(1)
$$

Fig. $5(\mathrm{~b}-\mathrm{c})$ show the products prepared at $\mathrm{pH}=7$ and 11 , respectively. The appearance of oxygen suggests that the oxide compounds of copper(I) or(II) might be produced. As sodium hydroxide was added in the adjustment of solution $\mathrm{pH}$ to 7 and 11, copper(II) hydroxide was likely formed which led to the formation of copper oxide. The molecular structure of the oxide compounds at $\mathrm{pH} 7$ was further examined by XRD measurements and the resultant diffraction patterns were illustrated in Fig. 6. All the diffraction peaks were labeled and matched well to the standard diffraction pattern of $\mathrm{Cu}_{2} \mathrm{O}$ in the JCPDS file No. 05-0667 $7,18,19,21$. Based on these results, the reaction pathways were proposed as follows ${ }^{7}$.

$$
\begin{gathered}
\mathrm{Cu}^{2+}+\mathrm{e}^{-} \rightarrow \mathrm{Cu}^{+} \\
\mathrm{Cu}^{+}+\mathrm{OH}^{-} \rightarrow \mathrm{Cu}(\mathrm{OH}) \\
2 \mathrm{Cu}(\mathrm{OH}) \rightarrow \mathrm{Cu}_{2} \mathrm{O}+\mathrm{H}_{2} \mathrm{O}
\end{gathered}
$$




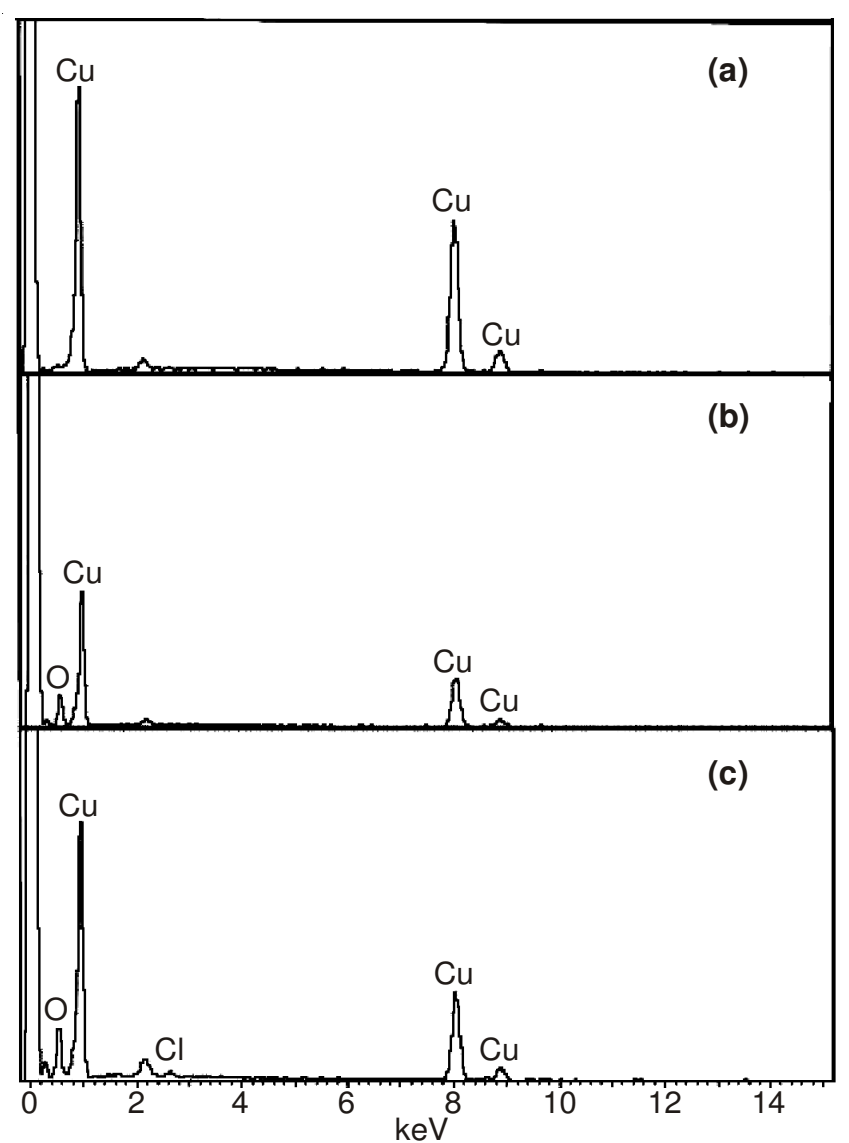

Fig. 5. EDX patterns of precipitates yielded from the reaction at (a) $\mathrm{pH} 3$ (b) $\mathrm{pH} 7$ (c) $\mathrm{pH} 11$

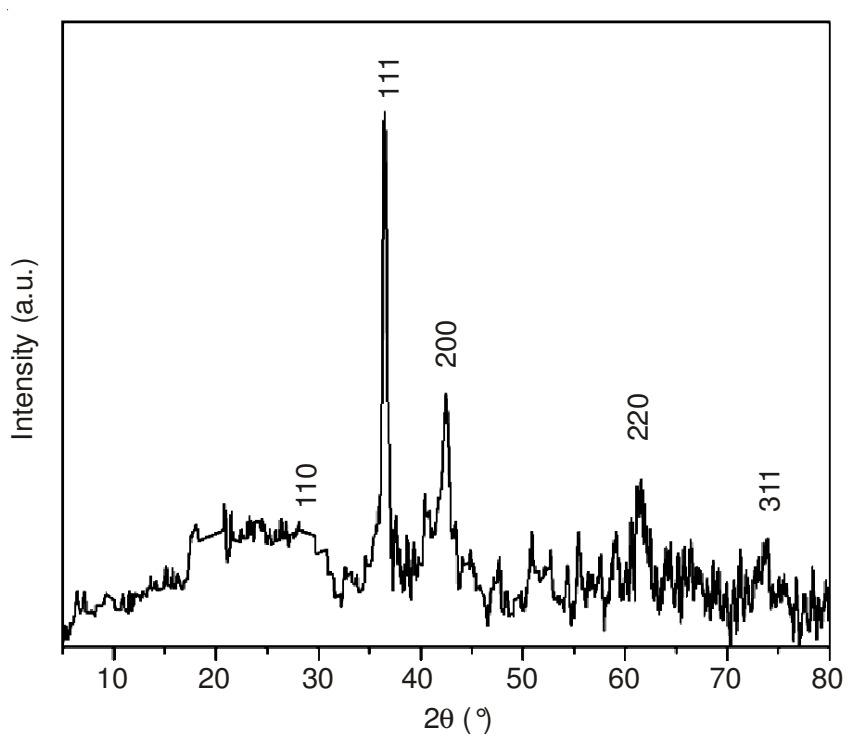

Fig. 6. XRD pattern of the prepared $\mathrm{Cu}_{2} \mathrm{O}$ hollow sphere

It is worth pointing out that the crystal morphology of the samples exhibited distinctions in two categories. First is the difference of the molecular structures. In the acid condition $(\mathrm{pH}=3)$, the reagent used for adjusting solution $\mathrm{pH}$ was sulfuric acid and the predominant product was pure copper. The growth of pure copper crystals is explained by the expansion of the geometrical lattices into a well-defined structures ${ }^{25}$ like triangles, dodecahedra and rods. In the other category, at the $\mathrm{pH}$ of 7 and 11 , the addition of hydroxide ions changed the reaction pathway and the products were copper oxide where copper and oxygen were bound with covalent bond in networks ${ }^{26}$. The different morphology of the products at $\mathrm{pH}=$ 7 and 11 are attributed to the different amounts of sodium hydroxide added. At neutral conditions, the added hydroxide ion is sufficient to form copper oxide nuclei which grew into tiny nanocrystals. After the coalescence of nanocrystalline grains, the spherical walls of the crystal are developed. At $\mathrm{pH}$ $=11$, the increasing amount of hydroxide ions led to an increasing rate of reaction ${ }^{12}$. The crystals grow rather uncontrollably into a network of crystals of a variety shapes. Therefore, many irregular tiny crystals including octahedra and spheres are yielded. This observation agrees well with results reported by He and co-worker?

$\mathrm{Cu}_{2} \mathrm{O}$ from PONPE-7, PONPE-9 and PONPE-12 template: Another series of experiments were carried out to synthesize $\mathrm{Cu}_{2} \mathrm{O}$ with the assistance of PONPE-7 and PONPE12, instead of PONPE-9, at neutral pH. Fig. 7 shows $\mathrm{Cu}_{2} \mathrm{O}$ produced at $\mathrm{pH}=7$ in the presence of PONPE-7 (a) and PONPE-9 (b) and PONPE-12 (c). It can be seen that that rather spherical $\mathrm{Cu}_{2} \mathrm{O}$ particles were produced with a comparable size. These results show that the ethylene oxide units in PONPE exerted minimal effects on the spherical morphology. However, the best hollow spheres were produced with the assistance of PONPE-9. It is therefore believed that the length of the ethylene oxide units may affect the rate of cluster growth which was responsible for the complete and/or incomplete fit among the tiny nanocrystals. This assumption requires deeper and more experimental studies.

$\mathrm{Cu}_{2} \mathrm{O}$ templated by other surfactants: Our success of the synthesizing $\mathrm{Cu}_{2} \mathrm{O}$ hollow spheres with the assistance of PONPE-9 motivated us to study other common surfactants. We repeated the experiments by substituting PONPE-9 with three other surfactants, SDS, AOT and CTAB. The SEM images of the corresponding precipitates are shown in Fig. 8. Fig. 8ab show the morphology of the products with SDS and AOT which are in the shape of cubes and octahedra, respectively. Both morphologies were also found in the reports of Kuo and coworkers $^{10}$, and Luo and coworkers ${ }^{11}$. The spherical shape was also found in the reaction mediated by CTAB in Fig. 8(c). The spherical morphology produced in the presence of CTAB was in accordance with the report of Xu et al..$^{20}$ who controlled the spherical shape of $\mathrm{Cu}_{2} \mathrm{O}$ with $\mathrm{CTAB}$ at $60^{\circ} \mathrm{C}$. However, in our reaction process, we found inconsistent results with the CTAB template in repeated experiments. In some batches we observed products of sheet-like and unidentified morphologies. It is likely that this inconsistency was due to the small variation of solution $\mathrm{pH}$ which may impact adsorption of cationic surfactants like $\mathrm{CTAB}^{27,28}$. Nonetheless, these results suggested that $\mathrm{CTAB}$ might not suitable in the current procedure. In contrast, repeated experiments using PONPE have shown good consistency suggesting $\mathrm{pH}$ tolerance of this surfactant around neutral $\mathrm{pH}$. Then, the usage of PONPE as a shape controller should provide more convenience in operation and enable large scale production. One more advantage of using PONPE was the lower surfactant concentration required in the process. In our experiment, the surfactant concentration was about $0.05 \mathrm{M}$ 

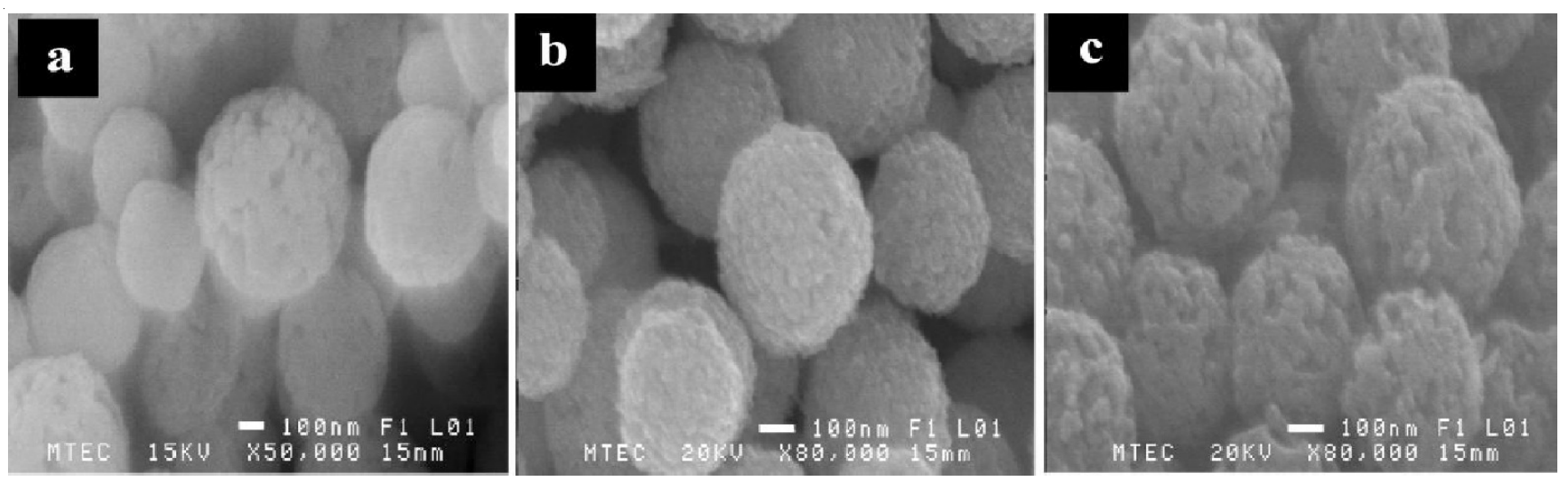

Fig. 7. SEM pictures of prepared $\mathrm{Cu}_{2} \mathrm{O}$ hollow sphere templated by (a) PONPE-7 (b) PONPE-9 (c) PONPE-12
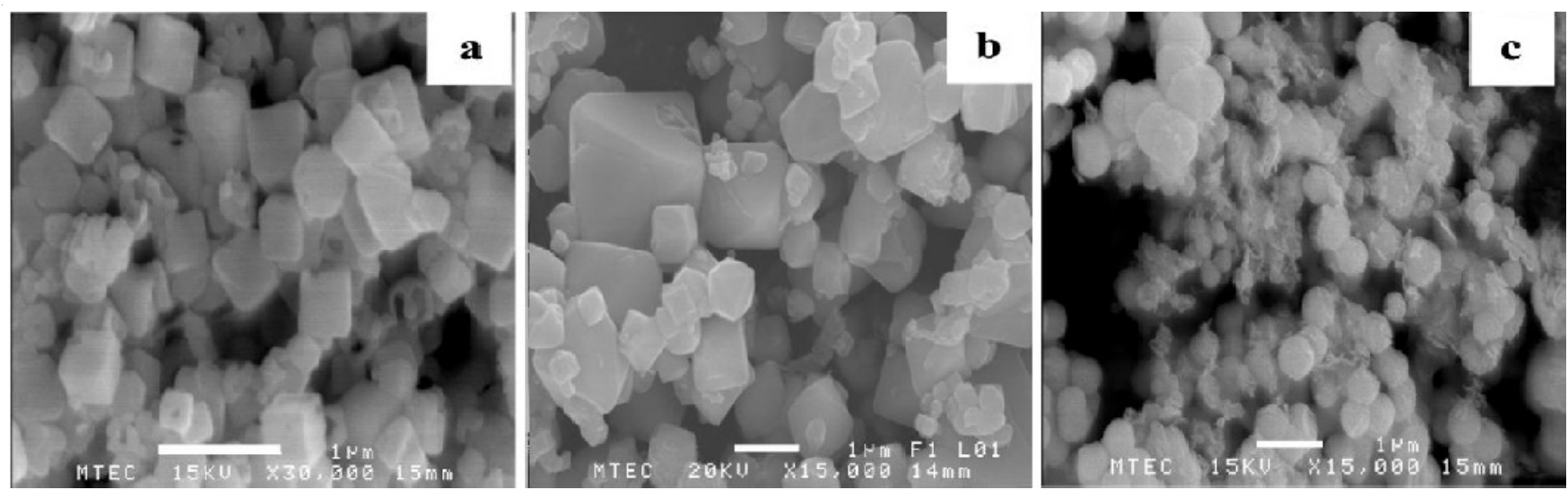

Fig. 8. SEM pictures of $\mathrm{Cu}_{2} \mathrm{O}$ produced in the presence of (a) SDS (b) AOT (c) CTAB

which was only half of the CTAB concentration used by Xu and co-workers. It should be mentioned here that lower surfactant concentration leads to shorter reaction time and less chemicals consumed in the washing process. Hence, PONPE is a promising soft template for the synthesis of hollow sphere crystals under mass production.

Proposed mechanism for self-assembly $\mathrm{Cu}_{2} \mathrm{O}$ hollow sphere: The possible mechanism for the PONPE-assisted production of hollow spheres of copper(I) oxide is proposed in Fig. $9^{20,29}$. We proposed the selective adsorption of PONPE at the air-water interface surrounding the surface of the surfactant bubbles produced by sonication. The stabilized bubbles lead to the formation of micelle-like cluster with a hollow interior. The polar head of the surfactants function as a specific site for the growth of $\mathrm{Cu}_{2} \mathrm{O}$ via a reduction process.<smiles>Nc1ccc(OCCO)cc1</smiles>

PONPE-9

Polyoxyethylene(9)nonylphenylether

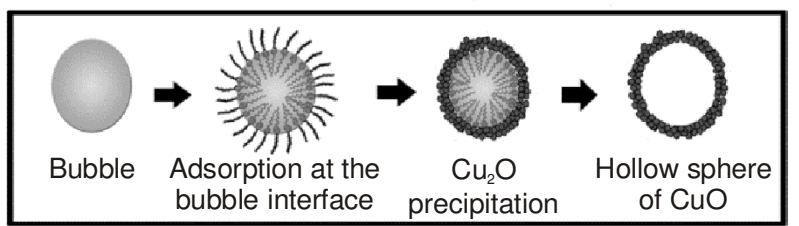

Fig. 9. Schematic for formation of $\mathrm{Cu}_{2} \mathrm{O}$ hollow structure in the presence of PONPE under sonication
With the reaction going on, crystal growth and solidification at the interface was transcriptive imprinting of the template morphology leading to the formation of hollow structured materials. In the washing step, surfactants could be washed off through the porous surface from inside the sphere and left the hollow sphere structure of the product.

\section{Conclusion}

In summary, $\mathrm{Cu}_{2} \mathrm{O}$ hollow spheres were successfully prepared in an aqueous solution of copper sulphate via a simple hydrothermal method with the assisting of polyoxyethylene nonylphenyl ether surfactants and no rigid template. Ascorbic acid was used as the reductant. The solution $\mathrm{pH}$ was adjusted to 7 and self assembly of hollow spheres of $\mathrm{Cu}_{2} \mathrm{O}$ was produced within a couple of hours at a low temperature of $40{ }^{\circ} \mathrm{C}$. No more reaction process was required. The diameter of the $\mathrm{Cu}_{2} \mathrm{O}$ particles was 0.6-1.5 $\mu \mathrm{m}$. The method was simple and reproducible. Thus, this would be a promising procedure for the synthesis of $\mathrm{Cu}_{2} \mathrm{O}$ hollow spheres in large scale.

\section{ACKNOWLEDGEMENTS}

The authors expressed their thanks to Rangsit Research Institute, Rangsit University, Thailand, for their financial support under the grant number RI 03/2012. Sincere thanks are due to Professor Shaowei Chen, Department of Chemistry and Biochemistry, University of California, Santa Cruz for his value suggestions. 


\section{REFERENCES}

1. Y. Cao, J. Fan, L. Bai, F. Yuan and Y. Chen, Cryst. Growth Des., 10, 232 (2010).

2. B. White, M. Yin, A. Hall, D. Le, S. Stolbov, T. Rahman, N. Turro and S. O'Brien, Nano Lett., 6, 2095 (2006).

3. C.H. Kuo and M.H. Huang, Nano Today, 5, 106 (2010).

4. J.L. Cuya Huaman, K. Sato, S. Kurita, T. Matsumoto and B. Jeyadevan, J. Mater. Chem., 21, 7062 (2011).

5. J.T. Zhang, J.F. Liu, Q. Peng, X. Wang and Y.D. Li, Chem. Matter., 18, 867 (2006).

6. T.M.D. Dang, T.T.T. Le, E. Fribourg-Blanc and M.C. Dang, Adv. Nat. Sci.: Nanosci. Nanotechnol., 2, 015009 (2011).

7. P. He, X. Shen and H. Geo, J. Colloid Interf. Sci., 284, 510 (2005).

8. M.H. Kim, B. Lim, E.P. Lee and Y. Xia, J. Mater. Chem., 18, 4069 (2008).

9. H.M. Song, D.H. Anjum, R. Sougrat, M.N. Hedhili and N.M. Khashab, J. Mater. Chem., 22, 25003 (2012).

10. H. Liu, W. Miao, S. Yang, Z. Zhang and J. Chen, Cryst. Growth Des., 9, 1733 (2009).

11. F. Luo, D. Wu, L. Gao, S. Lian, E. Wang, Z. Kang, Y. Lan and L. Xu, J. Cryst. Growth, 285, 534 (2005).

12. D. Wang, D. Yu, M. Mo, X. Liu and Y. Qian, J. Colloid Interf. Sci., 261, 535 (2003).

13. H. Zhang, X. Ren and Z. Cui, J. Cryst. Growth, 304, 206 (2007).

14. H. Xu, W. Wang and W. Zhu, J. Phys. Chem. B, 110, 13829 (2006).
15. L.L. Hung, C.K. Tsung, W. Huang and P. Yang, Adv. Mater, 22, 1910 (2010).

16. J.C. Park, J. Kim, H. Kwon and H. Song, Adv. Mater, 21, 803 (2009).

17. L. Xu, X. Chen, Y. Wu, C. Chen, W. Li, W. Pan and Y. Wang, Nanotechnology, 17, 1501 (2006).

18. H.J. Liu, Y.H. Ni, F. Wang, G. Yin, J.M. Hong, Q. Ma and Z. Xu, Colloid Surf. A, 235, 79 (2004).

19. Y. Chang, J.J. Teo and H.C. Zeng, Langmuir, 21, 1074 (2005).

20. Y. Sui, Y. Zhang, W. Fu, H. Yang, Q. Zhao, P. Sun, D. Ma, M. Yuan, Y. Li and G. Zou, J. Crystal Growth, 311, 2285 (2009).

21. J. Xu, C. Yao and Y. Wei, Asian J. Chem., 22, 6537 (2010).

22. H. Xu and W. Wang, Angew. Chem. Int. Ed., 46, 1489 (2007).

23. H. Zhang, Q. Zhu, Y. Zhang, Z. Li and B. Yu, Adv. Funct. Mater, 17, 2766 (2007).

24. Y. Park, W. Shin and J. Lee, CrystEngComm., 14, 4612 (2012).

25. Y. Xia, Y. Xiong, B. Lim and S.E. Skrabalak, Angew. Chem. Int. Ed., 48, 60 (2009)

26. T.D. Nguyen and T.O. Do, in ed.: Y. Masuda, Nanocrystal, In Tech Publisher, pp. 55-84 (2011); Available at http://www.intechopen.com/ books

27. R. Atkin, V.S.J. Craig and S. Biggs, Langmuir, 16, 9374 (2000).

28. T.P. Goloub, L.K. Koopal, B.H. Bijsterbosch and M.P. Sidorova, Langmuir, 12, 3188 (1996).

29. X.W. Lou, L.A. Archer and Z. Yang, Adv. Mater, 20, 3987 (2008). 